The Astrophysical Journal, 627:L105-L108, 2005 July 10

(C) 2005. The American Astronomical Society. All rights reserved. Printed in U.S.A.

\title{
VLA IMAGING OF THE INTRIGUING H I CLOUD HIJASS J1021+6842 IN THE M81 GROUP
}

\author{
FABIAN WALTER \\ Max-Planck-Institute for Astronomy, Königstuhl 17, D-69117 Heidelberg, Germany; walter@mpia-hd.mpg.de \\ Evan D. SKillman \\ Astronomy Department, University of Minnesota, 116 Church Street SE, Minneapolis, MN 55455; skillman@astro.umn.edu \\ AND \\ ELIAS BRINKS \\ Centre for Astrophysics Research, University of Hertfordshire, College Lane, Hatfield, Hertfordshire, AL10 9AB, UK; and INAOE, Apartado Postal 51, \\ Puebla, Pue 72000, Mexico; ebrinks@ star.herts.ac.uk \\ Received 2004 December 16; accepted 2005 May 31; published 2005 June 22
}

\begin{abstract}
We present VLA H i $21 \mathrm{~cm}$ observations of HIJASS J1021+6842, which has been discovered in the direction of the M81 Group. Our synthesis imaging reveals that the H I is distributed over a larger angular extent and velocity range than the single-dish discovery observations. Assuming that HIJASS J1021+6842 is at the distance of the M81 Group, we detect $1.5 \times 10^{8} M_{\odot}$ of $\mathrm{H}$ I distributed over as much as $30 \mathrm{kpc}$, i.e., substantially larger than the biggest dwarf galaxies in the same group. At the depth of our imaging, the $\mathrm{H}$ I appears to be confined to at least seven clouds. Peak H I column densities are $\sim 1.8 \times 10^{20}$ atoms $\mathrm{cm}^{-2}$, which is well below the canonical star formation threshold of $\sim 10^{21}$ atoms $\mathrm{cm}^{-2}$ and therefore consistent with the fact that no optical counterpart has as yet been identified. A gradient in velocity is observed across the extent of the detected $\mathrm{H} \mathrm{I}$; assuming that the object is gravitationally bound we derive a dynamical mass of $7 \times 10^{9} M_{\odot}$ and a dark-to-luminous mass ratio of $>10$. Alternatively, a tidal origin may also result in the observed velocity gradient, which would lead to a considerably lower dynamical mass. Given the above properties and the absence of evidence of a stellar population, HIJASS J1021+6842 is unique amongst the other systems in the M81 Group.
\end{abstract}

Subject headings: galaxies: clusters: general — galaxies: dwarf — galaxies: formation galaxies: individual (HIJASS J1021+6842) — radio lines: galaxies

\section{INTRODUCTION}

The nearby M81 Group provides an ideal test bed for studies of dwarf galaxies and the effects of interactions between galaxies. Thanks to its proximity, it is one of the best studied groups, both regarding the interaction between its three luminous galaxies (M81, M82, and NGC 3077, e.g., Yun et al. 1994), and the studies of individual group members, notably several of the dwarf galaxies (e.g., Puche et al. 1992; Walter \& Brinks 1999; Walter et al. 2002; Ott et al. 2001).

Boyce et al. (2001) recently conducted a blind H I survey of the M81 Group using a multibeam receiver on the $76 \mathrm{~m}$ Lovell telescope with a resultant beam size of $\approx 12^{\prime}$. Interestingly, only one new object was discovered in their observations, HIJASS J1021+6842, which is the subject of this letter. This implies that the census of galaxies with $\mathrm{H}$ I gas in the M81 Group must be nearly complete down to $\mathrm{H}$ I masses of order $10^{7} M_{\odot}$.

HIJASS J1021+6842 lies at an angular distance of 105' (or a minimum separation of $110 \mathrm{kpc}$ at the distance of M81) from IC 2574 . The proximity in sky position and radial velocity between IC 2574 and HIJASS J1021+6842 implies that HIJASS J1021+6842 is a probable member of the M81 Group at a distance of $\sim 4 \mathrm{Mpc}$ and a possible companion of IC 2574 (Boyce et al. 2001; Karachentsev et al. 2002). Boyce et al. (2001) noted the lack of an optical counterpart for HIJASS $\mathrm{J} 1021+6842$ in the second-generation red Digital Sky Survey and speculated that it may be either a very low surface brightness companion of IC 2574 or the debris of a tidal encounter between IC 2574 and one of the galaxies around M81.

Thus, HIJASS J1021+6842 is an intrinsically interesting object, and potentially a key object for understanding the process of galaxy formation from tidal debris. The present higher resolution $\mathrm{H}$ I observations provide a requisite first step to understanding the true nature of this object.

\section{OBSERVATIONS AND ANALYSIS}

We used the NRAO ${ }^{1}$ Very Large Array (VLA) to obtain $\mathrm{H}$ I spectral line observations of HIJASS J1021+6842 in the D configuration (integration time: 106 minutes) and in the C configuration (148 minutes) in 2004. We observed $0841+708$ as the complex gain calibrator and $0542+498$ (3C 147) and $1331+305$ (3C 286) as flux and bandpass calibrators. The spectra consisted of 128 channels with a width of $5.2 \mathrm{~km} \mathrm{~s}^{-1}$ (after online Hanning smoothing). The data were analyzed using standard calibration/mapping tasks in the AIPS software package. Four channels near velocities of $0 \mathrm{~km} \mathrm{~s}^{-1}$ were contaminated by Galactic foreground emission (see Fig. 1). To calculate the integrated H I map (moment 0) we carefully inspected and blanked consecutive channel maps to remove the extended Galactic emission from the data cube. In doing so we are helped by the fact that smoothly distributed emission, such as that due to foreground Galactic $\mathrm{H} \mathrm{I}$, is filtered out in interferometric observations.

Given the intrinsically faint $\mathrm{H}$ I emission of HIJASS $\mathrm{J} 1021+6842$, images were made with a UV taper of $5.3 \mathrm{k} \lambda$ to emphasize weak, extended structures. This resulted in a beam size of $60^{\prime \prime} \times 52^{\prime \prime}\left(60^{\prime \prime}=1.17 \mathrm{kpc}\right)$ and an $\mathrm{rms}$ of $0.8 \mathrm{mJy}$ beam $^{-1}$ per channel. Given the considerable extent of the H I emission, it was important to correct for primary beam attenuation in all subsequent, quantitative analysis.

\footnotetext{
${ }^{1}$ The National Radio Astronomy Observatory is a facility of the National Science Foundation operated under cooperative agreement by Associated Universities, Inc.
} 


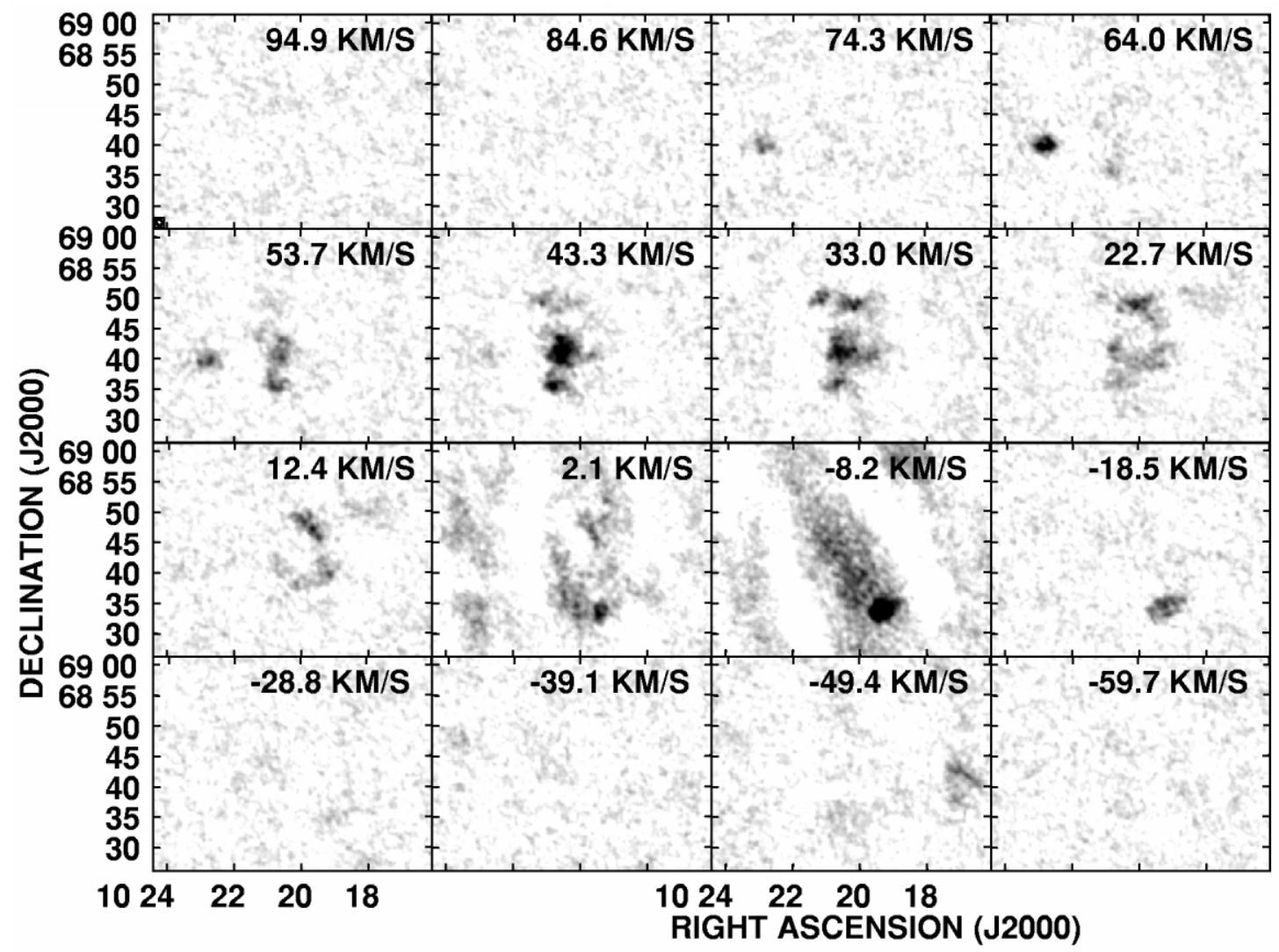

FIG. 1.-H I channel maps of HIJASS J1021+6842 (every other channel is shown, primary beam attenuation has not been applied). The gray scale displays a range from 0 to $9 \mathrm{mJy}_{\text {beam }}{ }^{-1}$. The beam size $\left(60^{\prime \prime} \times 52^{\prime \prime}\right)$ is indicated in the lower left of the top left panel. Note that the channel maps at around $0 \mathrm{~km} \mathrm{~s}{ }^{-1}$ are affected by large-scale Galactic H I emission.

\section{H I DISTRIBUTION AND KINEMATICS}

\subsection{H I Distribution}

Figure 1 shows a mosaic of 12 channel maps (before primary beam correction) stepped at every other channel. This mosaic shows that $\mathrm{H} \mathrm{I}$ is detected from $\sim-50$ to $\sim+70 \mathrm{~km} \mathrm{~s}^{-1}$ in radial velocity, which is a larger range in velocity than the FWHM of $\approx 50 \mathrm{~km} \mathrm{~s}^{-1}$ reported by Boyce et al. (2001). It is likely that the synthesis observations have allowed us to overcome confusion with Galactic $\mathrm{H}$ I emission, enabling us to detect $\mathrm{H} \mathrm{I}$ at velocities near $0 \mathrm{~km} \mathrm{~s}^{-1}$ and lower velocities. Additionally, the higher resolution imaging shows that the single source discovered by Boyce et al. (2001) breaks up into several regions of emission. There is a radial velocity gradient with positive velocities in the eastern components and negative velocities in the western components. What is particularly striking about these images is the fact that the $\mathrm{H}$ I is extended over more than $30 \mathrm{kpc}$, which makes HJJASS J1021+6842 bigger than even the most massive dwarf galaxies in the M81 Group (e.g., IC 2574: Walter \& Brinks 1999; Ho II: Puche et al. 1992).

Figure 2 shows a map of the total $\mathrm{H}$ I column density of HIJASS J1021+6842. We identify seven regions of emission (labeled I-VII in Fig. 2). The maximum H I column density is found in region VI and corresponds to $1.8 \times 10^{20}$ atoms $\mathrm{cm}^{-2}$. Region III is a marginal detection and needs follow-up observations.

The total H I emission shown in Figure 2 corresponds to a total H I mass of $1.5 \times 10^{8} M_{\odot}$. This is considerably larger than the value reported by Boyce et al. (2001). We attribute the difference to the fact that most of the emission is situated in small clumps (compared to the Lovell beam, FWHM = $\sim 12^{\prime}$ ), that the area over which the clumps are spread out is larger than the size of the Lovell beam, and that HIJASS $\mathrm{J} 1021+6842$ extends across velocities containing Galactic $\mathrm{H}$ I emission. The $\mathrm{H}$ I masses of the individual regions are listed in the caption of Figure 2.

\subsection{H I Kinematics and Dynamical Mass Estimate}

The channel maps (Fig. 1) show that there is a velocity gradient across the $\mathrm{H}$ I extent of HIJASS J1021+6842 extending from region I (near $70 \mathrm{~km} \mathrm{~s}^{-1}$ ) to region VII $\left(-50 \mathrm{~km} \mathrm{~s}^{-1}\right.$ ). The velocities at which the individual regions appear brightest in the channel maps are given in Figure 2 (right).

One interpretation of the velocity gradient is that the emission is gravitationally bound and that the velocity gradient can be interpreted as being due to a (broken) annulus in rotation. Under this assumption we find for a radius of $15 \mathrm{kpc}$ and a $V_{\max }$ of 40 $\mathrm{km} \mathrm{s}^{-1}$ a minimum dynamical mass of $5.5 \times 10^{9} M_{\odot}$. The resulting ratio of dynamical over detected mass (or $M_{\mathrm{dyn}} / M_{\mathrm{H}}$ ) is $>10$ for the ensemble. This would be a large ratio that is higher than is typically observed in lower luminosity dwarf irregular galaxies (Skillman 1996). We stress, however, that a tidal origin may also result in the observed velocity gradient, which would imply a significantly lower dynamical mass.

\section{CONSTRAINTS ON THE TRUE NATURE OF HIJASS J1021+6842}

\subsection{Lack of an Optical Counterpart}

As noted by Boyce et al. (2001), there is no obvious optical counterpart to HIJASS J1021+6842, which is detected on images 


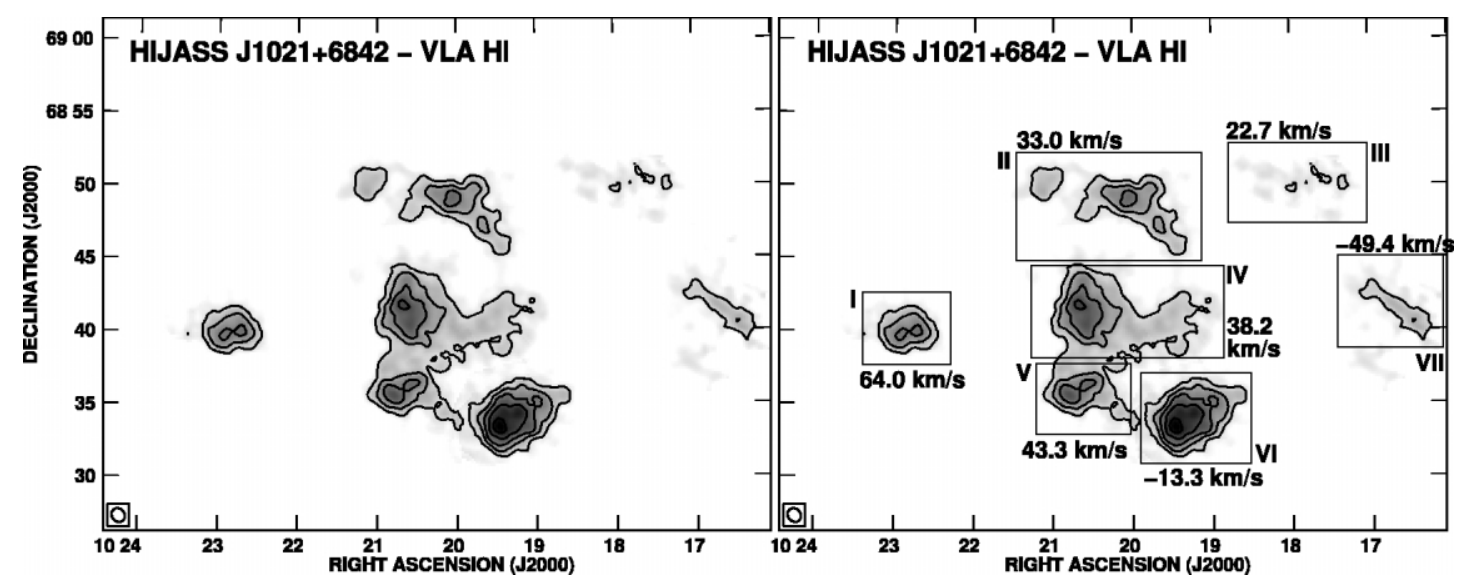

FIG. 2.-Total H I column density image of HIJASS J1021+6842 (corrected for primary beam attenuation). The field size is identical to that of Fig. 1. The beam size $\left(60^{\prime \prime} \times 52^{\prime \prime}\right)$ is indicated in the lower left. Contours are plotted at $4,7,10,13$, and $16 \times 10^{19}$ atoms $\mathrm{cm}^{-2}$. The total $\mathrm{H}$ I mass is $1.5 \times 10^{8} M_{\odot}$. The boxes in the right-hand panel indicate regions with coherent structures in position-velocity space. Masses for the individual regions are as follows (in units of $10^{7} M_{\odot}$ ): $\mathrm{I}=1.3$; $\mathrm{II}=2.6 ; \mathrm{III}=0.8 ; \mathrm{IV}=4.1 ; \mathrm{V}=1.5 ; \mathrm{VI}=3.0 ; \mathrm{VII}=1.1$. The numbers adjacent to the boxes give the velocities at which the individual regions appear brightest in the channel maps. Note an apparent gradient in velocity ranging from around $70 \mathrm{~km} \mathrm{~s}^{-1}$ (region I) to $-50 \mathrm{~km} \mathrm{~s}^{-1}$ (region VII).

of the DSS. In addition to this, the M81 Group has been the subject of many very thorough searches for small and low surface brightness members (e.g., van den Bergh 1959; Karachentseva 1968; Boerngen \& Karachentseva 1982; Caldwell et al. 1998; Karachentseva \& Karachentsev 1998). These searches reached faint surface brightness levels and lead to the discovery of dwarfs as faint as $m_{V} \sim 18$, corresponding to $M_{V} \sim-10$ at the distance of the M81 Group (thus reaching down to the extreme low-mass end of the luminosity function). As yet, none of these searches have come up with candidate counterparts for HIJASS J1021+ 6842 (e.g., Caldwell et al. 1998). One possible concern is the presence of a bright star in the field (HD 89343, R.A. = $10^{\mathrm{h}} 21^{\mathrm{m}} 03.3, \quad$ decl. $\left.=+68^{\circ} 44^{\prime} 52^{\prime \prime} \quad(\mathrm{J} 2000.0), \quad V=6 \mathrm{mag}\right)$, which may limit the local sensitivity to low surface brightness optical emission.

It should be noted, though, that the $\mathrm{H}$ I distribution is so different from the other M81 Group dwarfs that perhaps one would not necessarily expect stars to be associated with this system. Normally, star formation is associated with H I column densities above a certain threshold (e.g., Kennicutt 1989). Empirically this threshold hovers around $10^{21}$ atoms $\mathrm{cm}^{-2}$ for dwarf irregular galaxies. The fact that no optical counterpart has been found appears to be consistent with this threshold. Clearly, deep optical follow-up observations are needed to determine at higher confidence whether stars are associated with HIJASS $\mathrm{J} 1021+6842$.

Another possible explanation for the lack of stars in HIJASS $\mathrm{J} 1021+6842$ is the possibility that we are seeing the early stages of formation of a new galaxy. This could either be formation from a primordial cloud or formation from tidal debris as discussed, e.g., by Makarova et al. (2002) or Bournaud et al. (2004 and references therein). Given the relatively dense environment of the central M81 triplet, the position of HIJASS $\mathrm{J} 1021+6842$ seems to be an unlikely location for the contemporary precipitation of a primordial galaxy. If HIJASS $\mathrm{J} 1021+6842$ is tidal debris (perhaps from the outer parts of one of the larger galaxies in the M81 Group), then the small velocity differences between its components allow for the possibility that the entire system is gravitationally bound, which could potentially lead to future concentration and the formation of stars.

\subsection{Group Membership}

Since HIJASS J1021+6842 was discovered in a survey of the M81 Group, it is natural to assume that it is a member of that group. Nonetheless, HIJASS J1021+6842 is an unusual system and, before assuming group membership, it is important to consider evidence in favor or against this assumption. An overview of the relative locations of the M81 Group members in the plane of the sky is presented in Karachentsev et al. (2002, their Fig. 1). The systemic velocity of $\approx 30 \mathrm{~km} \mathrm{~s}^{-1}$ is certainly consistent with membership in the M81 Group (Karachentsev et al. 2002). In fact, it is only offset from M81 by 2.3 in angle (corresponding to a minimum distance of $\sim 140 \mathrm{kpc}$ ) and 40 $\mathrm{km} \mathrm{s}^{-1}$ in radial velocity. In addition, HIJASS J1021+6842 is situated very close to the M81 Group member IC 2574 both in position (projected distance: $110 \mathrm{kpc}$ ) and velocity $(\Delta v=30$ $\mathrm{km} \mathrm{s}^{-1}$ ). This may suggest that IC 2574 and HIJASS J1021+ 6842 are companions, a scenario that is tentatively supported by the HIJASS observations, which indicate a low column density $\mathrm{H}$ I bridge connecting them.

What is the probability that this object is part of the Local Group rather than the M81 Group? In that case, HIJASS $\mathrm{J} 1021+6842$ would likely be classified as a high-velocity cloud (HVC). There are several problems with such a hypothesis, however. A strong argument against it is its fairly high column density, of order $10^{20}$ atoms $\mathrm{cm}^{-2}$, which is at least an order of magnitude higher than what is found even in the Magellanic Stream (Putman et al. 2003). Given its angular size, HIJASS $\mathrm{J} 1021+6842$ would be classified as a compact HVC. Again, typical column densities for compact HVCs are at least an order of magnitude lower (de Heij et al. 2002). An additional strong argument arguing against HIJASS J1021 +6842 being an HVC is that typical HVCs in the direction of M81 have velocities in the range -150 to $-200 \mathrm{~km} \mathrm{~s}^{-1}$ (Wakker \& van Woerden 1997), i.e., at much lower velocities. We therefore conclude that HIJASS J1021+6842 is indeed associated with the M81 Group.

\section{CONCLUSIONS}

We present VLA H I $21 \mathrm{~cm}$ observations of HIJASS $\mathrm{J} 1021+6842$ discovered by Boyce et al. (2001) in the direction of the M81 Group. Given its location, column densities, and 
systemic velocity, HIJASS J1021 +6842 is very likely a member of the M81 Group. Perhaps the most striking result is that the $\mathrm{H} \mathrm{I}$ emission is distributed over an area of $30 \mathrm{kpc}$ in diameter, which is much larger than the extent of the largest dwarf members of the M81 Group. This, and the lack of detectable stars, make the HIJASS J1021+6842 system absolutely unique amongst the M81 Group members. Other H I clouds known to date that have no detected optical counterparts are the southwest clump of H I 1225+01 (Giovanelli \& Haynes 1989; Chengalur et al. 1995; Turner \& MacFadyen 1997), HIPASS J0731 -69 (Ryder et al. 2001), and H I clouds in Virgo (Davies et al. 2004; Minchin et al. 2005). We detect $1.6 \times$ $10^{8} M_{\odot}$ of $\mathrm{H}$ I distributed over roughly $30 \mathrm{kpc}$ in HIJASS $\mathrm{J} 1021+6842$. Peak H I column densities are of order $1.8 \times$ $10^{20}$ atoms $\mathrm{cm}^{-2}$, which is well below the empirical threshold for star formation activity to commence. This may explain why to date no optical counterpart has been identified.

The individual clouds that make up the system are either self-gravitating entities orbiting within their mutual gravitational potential, or the densest concentrations of a huge, $30 \mathrm{kpc}$ diameter, gently rotating, very low surface density cloud. Assuming that the entire complex is gravitationally bound, we derive a minimum dynamical mass of $5.5 \times 10^{9} M_{\odot}$, which would be more than an order of magnitude more massive than the luminous ( $\mathrm{H} \mathrm{I}$ ) mass. It should be stressed, though, that other scenarios, such as a past stripping event leading to the formation of tidal debris, may also result in the observed velocity structure (thus leading to a significantly lower dynamical mass of the system).

The present observations clearly demonstrate the uniqueness of HIJASS J1021+6842. Future high-sensitivity H I synthesis observations of HIJASS J1021+6842 and its surroundings and deep optical and UV imaging will be necessary to elucidate the true nature of this enigmatic object.

E. D. S. is grateful for partial support from a NASA LTSARP grant NAG5-9221 and the University of Minnesota. We thank our referee, Mike Disney, for useful comments that helped to improve the presentation of this Letter.

\section{REFERENCES}

Boerngen, F., \& Karachentseva, V. E. 1982, Astron. Nachr., 303, 189

Bournaud, F., Duc, P.-A., Amram, P., Combes, F., \& Gach, J.-L. 2004, A\&A, 425,813

Boyce, P. J., et al. 2001, ApJ, 560, L127

Caldwell, N., Armandroff, T. E., Da Costa, G. S., \& Seitzer, P. 1998, AJ, 115, 535

Chengalur, J. N., Giovanelli, R., \& Haynes, M. P. 1995, AJ, 109, 2415

Davies, J., et al. 2004, MNRAS, 349, 922

de Heij, V., Braun, R., \& Burton, W. B. 2002, A\&A, 392, 417

Giovanelli, R., \& Haynes, M. P. 1989, ApJ, 346, L5

Karachentsev, I. D., et al. 2002, A\&A, 383, 125

Karachentseva, V. E. 1968, Soob. Byurakan Obs. 39, 62

Karachentseva, V. E., \& Karachentsev, I. D. 1998, A\&AS, 127, 409

Kennicutt, R. C., Jr. 1989, ApJ, 344, 685

Makarova, L. N., et al. 2002, A\&A, 396, 473

Minchin, R., et al. 2005, ApJ, 622, L21
Ott, J., Walter, F., Brinks, E., Van Dyk, S. D., Dirsch, B., \& Klein, U. 2001, AJ, 122, 3070

Puche, D., Westpfahl, D., Brinks, E., \& Roy, J. 1992, AJ, 103, 1841

Putman, M. E., Staveley-Smith, L., Freeman, K. C., Gibson, B. K., \& Barnes, D. G. 2003, ApJ, 586, 170

Ryder, S. D., et al. 2001, ApJ, 555, 232

Skillman, E. D. 1996, in ASP Conf. Ser. 106, The Minnesota Lectures on Extragalactic Neutral Hydrogen, ed. E. D. Skillman (San Francisco: ASP), 208

Turner, N. J. J., \& MacFadyen, A. 1997, MNRAS, 285, 125

van den Bergh, S. 1959, Publ. David Dunlap Obs., 2, 147

Wakker, B. P., \& van Woerden, H. 1997, ARA\&A, 35, 217

Walter, F., \& Brinks, E. 1999, AJ, 118, 273

Walter, F., Weiss, A., Martin, C., \& Scoville, N. 2002, AJ, 123, 225

Yun, M. S., Ho, P. T. P., \& Lo, K. Y. 1994, Nature, 372, 530 